\title{
Sonificación de EEG para la clasificación de palabras no pronunciadas
}

\author{
Erick Fernando González-Castañeda, Alejandro Antonio Torres-García, Carlos \\ Alberto Reyes-García, Luis Villaseñor-Pineda \\ Instituto Nacional de Astrofísica, Óptica y Electrónica (INAOE), \\ Tonantzintla, Puebla, México \\ \{erick.gonzalezc,alejandro.torres,kargaxxi,villasen\}@inaoep.mx
}

\begin{abstract}
Resumen. Las interfaces cerebro-computadora (BCI) basadas en electroencefalograma (EEG) son una alternativa que pretende integrar a las personas con discapacidad motriz severa a su entorno. Sin embargo, éstas aún no son utilizadas en la vida cotidiana por lo poco intuitivas que son las fuentes electrofisiológicas para controlarlas. Para tratar este problema, se han realizado trabajos con el objetivo de clasificar las señales de EEG registradas durante el habla imaginada. En este trabajo se utilizó la técnica de sonificación de señales de EEG, la cual nos permite caracterizar la señal de EEG como una señal de audio. El objetivo es analizar si al aplicar el proceso de sonificación de la señal de EEG se puede discriminar o resaltar patrones que mejoren los resultados de clasificación de palabras no pronunciadas. Para ello se procesó la señal con y sin sonificación. Se obtuvieron los resultados de los 4 canales más cercanos a las áreas de lenguaje de Broca y Wernicke. Los porcentajes de exactitud promedio para las señales sin aplicar sonificación y aplicando sonificación son $48.1 \%$ y $55.88 \%$, respectivamente, por lo que se pudo observar que el método empleado de sonificación de EEG mejora ligeramente los porcentajes de clasificación.
\end{abstract}

Palabras claves: Electroencefalogramas (EEG), interfaces cerebro-computadora (BCI), sonificación (sonification), habla imaginada (imagined speech/unspoken speech), random forest.

\section{Introducción}

En 2001 se presentó la "Clasificación Internacional del Funcionamiento, de la Discapacidad y de la Salud’' En ésta se establece que las personas con discapacidad son aquellas que tienen una o más deficiencias físicas, mentales, intelectuales o sensoriales y que al interactuar con distintos ambientes del entorno social pueden impedir su participación plena y efectiva en igualdad de condiciones a

\footnotetext{
${ }^{1}$ Clasificación Internacional del Funcionamiento, de la Discapacidad y de la Salud. Consultado el 10 de febrero de 2014. Disponible en http://www.conadis.salud. gob.mx/descargas/pdf/CIF_OMS_abreviada.pdf
} 


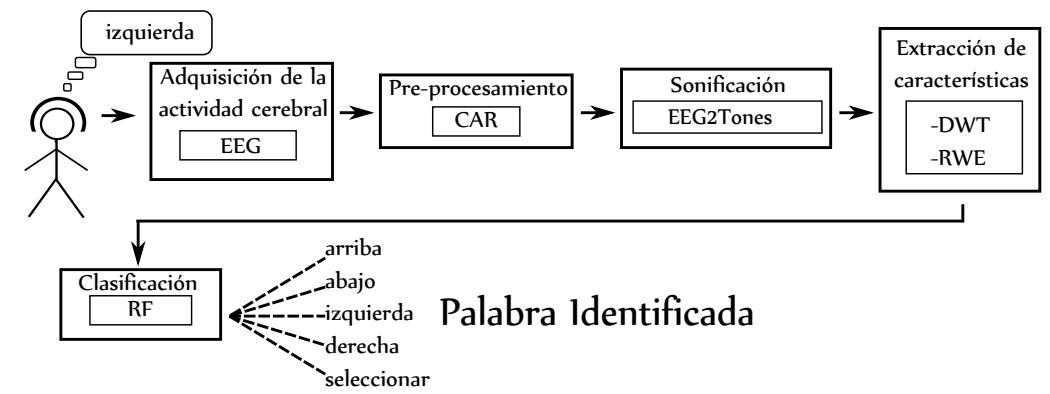

Fig. 1: Diagrama de la metodología seguida para clasificar señales de EEG registradas durante el habla imaginada. En los bloques internos se detalla el método propuesto. Además se ilustra a un individuo realizando la pronunciación imaginada de la palabra "izquierda" ante lo cual la tarea del método es inferir, con base a las señales de EEG registradas, cuál de las cinco palabras del vocabulario propuesto fue imaginada.

las demás. De acuerdo con el Informe mundial sobre la discapacidad 20112 de la Organización de las Naciones Unidas (ONU), en esta situación se estima que viven más de mil millones de personas en todo el mundo, esta cifra representa aproximadamente el $15 \%$ de la población mundial (según las estimaciones de la población mundial en 2010). Dentro de este porcentaje, se estima que casi 200 millones de personas experimentan dificultades considerables en su funcionamiento. Por ejemplo, discapacidades motrices severas como: la tetraplejía (cuadraplejía), la esclerosis lateral amiotrófica (ELA), la embolia (ictus cerebral), las lesiones de médula espinal o cerebral, la parálisis cerebral, las distrofias musculares, la esclerosis múltiple entre otros padecimientos. Estas discapacidades frecuentemente provocan que la persona no pueda controlar voluntariamente sus movimientos, incluyendo aquellos relacionados directa o indirectamente con la articulación del habla 6 628]. En consecuencia, una persona en estas condiciones está prácticamente aislada de su entorno.

En la búsqueda de un medio para integrar a la sociedad a las personas con discapacidad motriz severa, se ha explorado el uso de la actividad cerebral registrada mediante el electroencefalograma (EEG) para controlar dispositivos e interfaces. De forma general, una BCI puede ser vista como un sistema de reconocimiento de patrones donde el EEG es usado como la fuente primaria de información, un algoritmo de aprendizaje es usado para aprender una función de inferencia a partir del EEG, y por último, de acuerdo con la salida predicha por el algoritmo se ejecuta la acción deseada en el dispositivo a utilizar.

\subsection{Sonificación}

El concepto de sonificación (en inglés 'Sonification' o 'Auditory display'), se refiere al uso de sonido no hablado (non-speech audio) para transmitir informa-

\footnotetext{
${ }^{2}$ Informe mundial sobre la Discapacidad 2011. Consultado el 10 de febrero de 2014. Disponible en http://www.who.int/disabilities/world_report/2011/summary_ es.pdf
} 
ción [13. La sonificación es la contraparte de la visualización, es un método que en lugar de asignar posiciones específicas a los valores abstractos de acuerdo a las reglas uniformes en un diagrama, asigna sonidos a ellos, también siguiendo normas uniformes. En la sonificación se realiza la transformación de relaciones de datos en relaciones acústicas con el propósito de facilitar la comunicación y la interpretación. Podemos plasmar en sonido imágenes 2D, datos sismológicos e incluso información de datos estadísticos.

La sonificación de EEG ha sido utilizada para hacer análisis exploratorio de las señales de EEG, [12, para hacer composiciones musicales [5] o para hacer diagnóstico temprano de enfermedades neurológicas como la enfermedad de Alzheimer mediante la retroalimentación audible [2410]. Existen diversas técnicas para hacer sonificación de señales de EEG como: audificación, asignación por mapeo de parámetros, Sonificación basada en modelos [14], sonificación basada en modelado de bumps [21, entre otras.

\section{Problemática}

Una parte medular de la definición de BCI de Wolpaw 28] es cómo enviar los mensajes y comandos usando el EEG. La respuesta radica en los mecanismos neurológicos o procesos empleados por el usuario para generar las señales de control, denominadas fuentes electrofisiológicas. Las más utilizadas son los potenciales corticales lentos (SCP, por sus siglas en inglés), los potenciales P300, las imágenes motoras (ritmos sensoriales motrices mu y beta) y los potenciales evocados visuales (VEP, por sus siglas en inglés) [26]28].

Las fuentes electrofisiológicas descritas anteriormente (SCP, P300, imágenes motoras, y VEP) tiene dos grandes inconvenientes que repercuten en el posible uso de una BCI. El primero es el largo periodo de entrenamiento (algunas semanas o hasta meses) requerido para que un usuario pueda utilizar una BCI. Esto se debe que, estas fuentes son generadas por el usuario de forma poco consciente [17]. El segundo son las bajas tasas de comunicación (una sola palabra procesada, o menos, por minuto) que resultan insuficientes para permitir una interacción natural. Este último problema se debe a que cada una de estas fuentes requieren un "mapeo" o traducción al dominio del habla [6].

Recientemente, producto de los problemas descritos previamente, algunos trabajos tratan de utilizar los potenciales relacionados con la producción del habla, con diversos grados de éxito [6]. En estos trabajos, la fuente electrofisiológica es el habla imaginada (imagined speech), también referida como habla interna o habla no pronunciada (unspoken speech). De acuerdo con Wester [27], el término habla imaginada se refiere a la pronunciación interna, o imaginada, de palabras pero sin emitir sonidos ni articular gestos para ello. Es importante mencionar que, Denby [8] incluye a estos trabajos dentro de un área de investigación denominada interfaces de habla silente (SSI, por Silent Speech Interfaces) cuya finalidad es desarrollar sistemas capaces de permitir la comunicación "hablada" que tienen lugar cuando la emisión de una señal acústica entendible es imposible. Es importante remarcar que los trabajos que utilizan habla imaginada pueden 
dividirse, por la unidad de habla utilizada, en dos enfoques: palabras y sílabas.

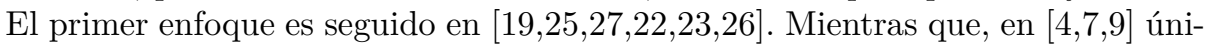
camente se tratan sílabas. La presente investigación tiene como objetivo aplicar un método de sonificación de EEG que permita obtener una retroalimentación audible de la señal cerebral durante la pronunciación imaginada de palabras y que además permita resaltar patrones que ayuden a un clasificador automático a mejorar los porcentajes de exactitud reportados en el trabajo de [23].

\section{Metodología}

El presente trabajo está compuesto de las siguientes etapas: Adquisición de la actividad cerebral, Preprocesamiento, Sonificación, Extracción de características y Clasificación. La metodología seguida en el trabajo se muestra de mejor manera en la Figura 1. Es importante mencionar que también se procesarán las señales de EEG sin la etapa de sonificación, de tal manera que pueda existir un marco de comparación con el trabajo descrito en [23].

\subsection{Adquisición de la actividad cerebral}

En esta etapa se utiliza el conjunto de datos de EEG registrados durante el habla imaginada utilizados en [23]. Este conjunto de datos se compone de las señales de EEG de 27 individuos cuyo idioma nativo es el español. Las señales de EEG se registraron utilizando el kit EPOC de EMOTIVC. Este kit es inalámbrico y consta de 14 canales (electrodos) de alta resolución (más las referencias CMS/DRL en las posiciones P3/P4, respectivamente) cuya frecuencia de muestreo es de $128 \mathrm{~Hz}$. Los nombres de los canales, de acuerdo con el sistema internacional 10-20, son: AF3, F7, F3, FC5, T7, P7, O1, O2, P8, T8, FC6, F4, F8, AF4 (ver Figura 2).

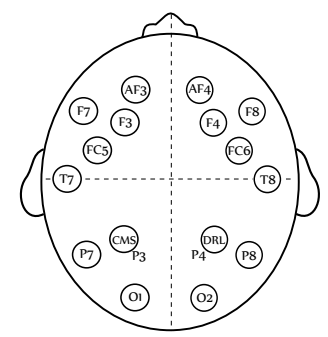

Fig. 2: Localización de los electrodos en el kit EPOC de Emotiv

Este conjunto de datos se registró por medio de un protocolo básico para adquirir las señales de EEG de cada individuo. El protocolo consistió en colocar a la persona cómodamente sentada con los ojos abiertos cerca de un escritorio, y con la mano derecha sobre el mouse de una computadora. Con un clic al mouse, 
el usuario delimitaba tanto el inicio como el fin de la pronunciación imaginada de alguna de las palabras del vocabulario reducido, compuesto de las siguientes cinco palabras en español: "arriba", "abajo", "izquierda", "derecha", y "seleccionar" (ver Figura 3). El objetivo detrás de este protocolo de adquisición es saber a priori en qué parte de la señal de EEG se deben buscar los patrones asociados con la pronunciación imaginada de la palabra indicada.

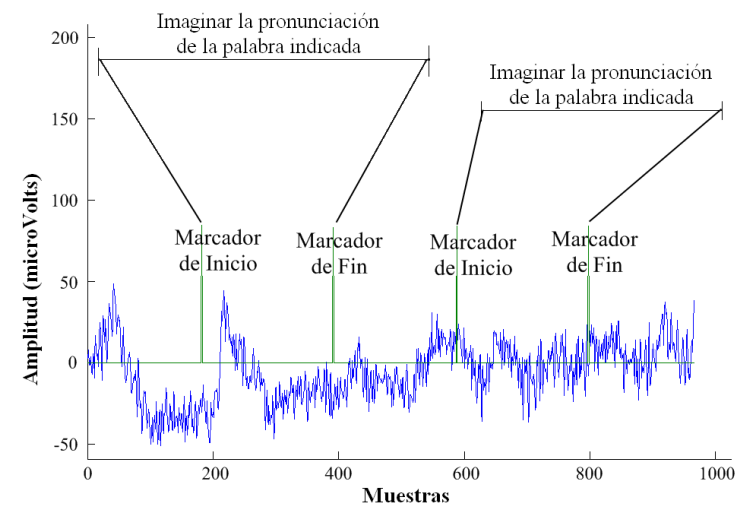

Fig. 3: Señal de EEG del canal F7 del individuo S1 mientras imagina la dicción de la palabra "Abajo" siguiendo el protocolo de adquisición de datos

Los segmentos de interés de las señales de EEG son aquellos que se encuentran entre los marcadores de inicio y fin, estos segmentos son denominados épocas (ventanas). Cada época tiene una duración variable como el habla normal (pronunciada). Además, la pronunciación imaginada de cada una de las cinco palabras fue repetida 33 veces consecutivas durante el registro del EEG, es decir, cinco bloques de 33 repeticiones por palabra. Antes de cada bloque se le indicó al individuo cuál es la palabra que debía pronunciar internamente. Todos las épocas de un mismo individuo fueron registradas en una única sesión (mismo día). Además, todas las sesiones se registraron en un laboratorio alejado de ruido audible externo y de ruido visual.

Es importante mencionar que al inicio del registro de las señales de EEG, se le indicó a la persona que evitara parpadear o realizar movimientos corporales mientras imaginaba la pronunciación de la palabra indicada, ya que después de cada marcador de fin podía tomarse un tiempo de descanso para dichos movimientos. Además, con la finalidad de que el individuo no supiera cuántas veces se repetiría una palabra, en la sala de experimentos, otra persona alejada del campo visual y guardando el debido silencio, se encargaba de realizar el conteo de repeticiones e indicaba cuando el individuo debía concluir. Esto con la finalidad de que el individuo no se distrajera contando el número de repeticiones ni se predispusiera a saber que le falta poco o mucho para concluir el experimento. 


\subsection{Preprocesamiento}

Las señales de EEG obtenidas son preprocesadas con el método de referencia promedio común (CAR, por sus siglas en inglés). Este método tiene como fin mejorar la relación señal a ruido de la señal de EEG. Básicamente, se busca quitar todo aquello que es común en todas las lecturas simultáneas de los electrodos. La CAR puede ser calculada mediante la resta del potencial entre cada electrodo y la referencia (el potencial promedio de todos los canales), se repite esto para cada instante de tiempo en la frecuencia de muestreo.

\subsection{Sonificación de la señal EEG}

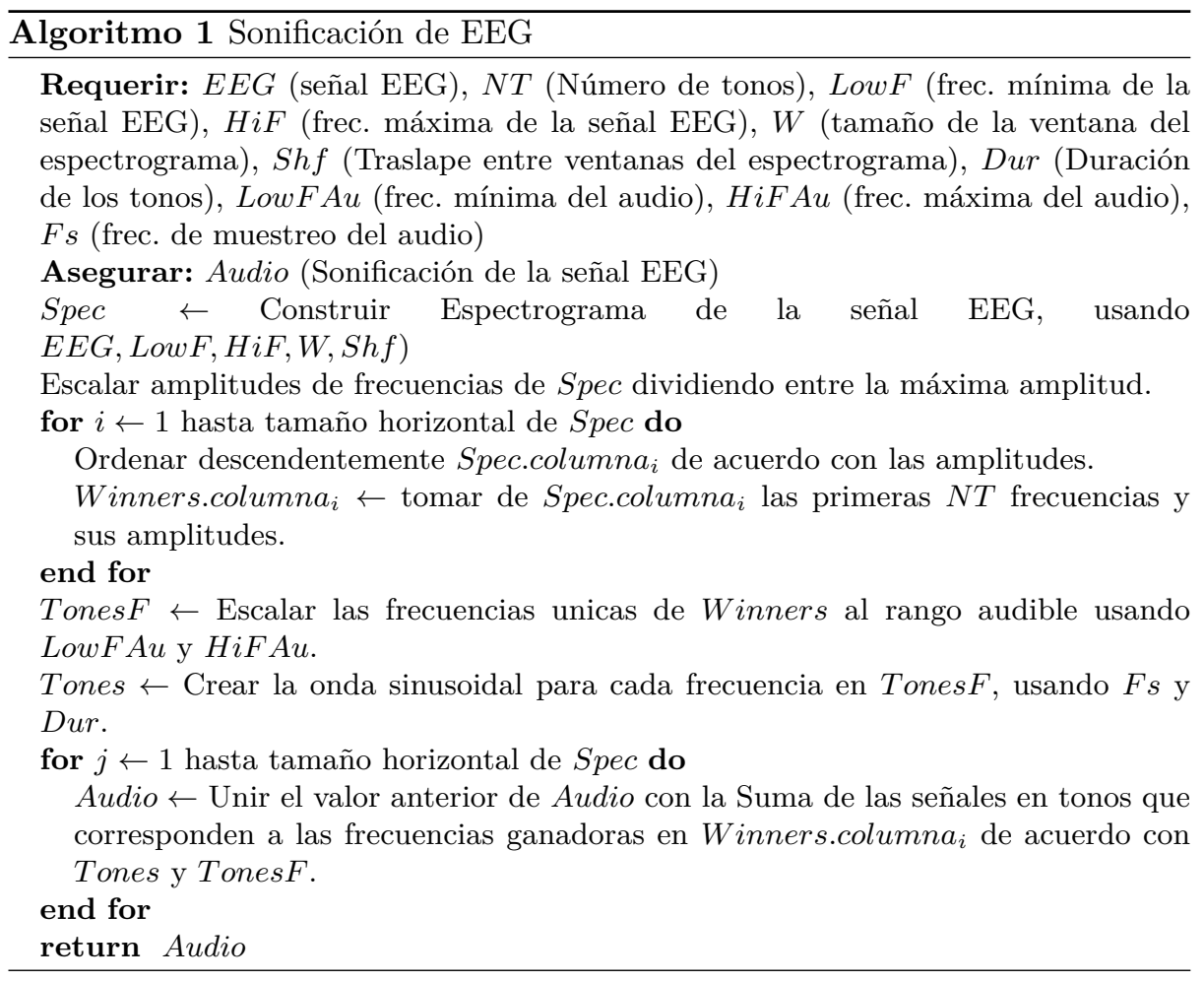

En el presente trabajo se utilizó la técnica de Sonificación 'EEG to tones' [1]. Esta técnica es un proceso de audificación, en la cual se escalan las frecuencias de EEG a frecuencias audibles. Esta técnica se basa en el espectrograma de la señal EEG de entrada, el cual es calculado usando la transformada rápida de Fourier $(F F T)$. De cada ventana del espectrograma se calculan las frecuencias dominantes de la señal de EEG. Las frecuencias dominantes son escaladas a tonos, los cuales representan una frecuencia en el rango audible. Al final cada 
conjunto de tonos dominante por ventana es unido para formar el audio de salida. El pseudocódigo que explica con más detalle el método utilizado es mostrado en el algoritmo 1 .

\subsection{Extracción de características}

Transformada wavelet discreta. En [15] se menciona que las características utilizadas en las BCI son no estacionarias ya que las señales de EEG pueden rápidamente variar con el tiempo. Además, estas características deben contener información del tiempo debido a que los patrones de actividad cerebral están generalmente relacionados a variaciones específicas del EEG en el tiempo. Lo anterior, hace necesaria una representación que considere eso.

Una técnica que permite modelar dichas variaciones, en el dominio tiempoescala, es la transformada wavelet discreta (DWT, por su siglas en inglés). La DWT provee una representación wavelet altamente eficiente mediante la restricción de la variación en la traslación y la escala, usualmente a potencias de dos. En ese caso, la DWT es algunas veces llamada transformada wavelet diádica.

El análisis DWT puede ser realizado usando un algoritmo piramidal rápido descrito en términos de bancos de filtros multi-tasa, es decir, aquellos donde se tiene más de una tasa de muestreo realizando conversiones mediante las operaciones de decimación e interpolación. La DWT puede ser vista como un banco de filtros con espacio de una octava entre ellos. Cada sub-banda contiene la mitad de las muestras de la frecuencia de la sub-banda vecina más alta. En el algoritmo piramidal la señal es analizada en diferentes bandas de frecuencias con diferentes resoluciones mediante la descomposición de la señal en una aproximación burda (coeficientes de aproximación) e información detallada (coeficientes de detalle). La aproximación burda es entonces adicionalmente descompuesta usando el mismo paso de descomposición wavelet. Esto se logra mediante un filtrado sucesivo de pasa-bajas y pasa-altas de la señal de tiempo, y un sub-muestreo. En [18 se muestra la información detallada de lo antes mencionado.

En el presente trabajo se aplica la transformada wavelet discreta a los archivos de audio resultantes de la sonificación. Se calcularon 6 niveles de descomposición usando la wavelet madre Daubechies de orden 20 (db20). Asimismo, para el caso de las señales de EEG que no fueron sonificadas, la DWT se calculó con 5 niveles de descomposición utilizando la Daubechies de segundo orden (db2), como se describe en 23 .

Como es evidente, el número de coeficientes wavelet en cada uno de los niveles variará dependiendo del tamaño de la señal de EEG delimitada entre los marcadores. Esto debido a que, de manera similar al habla convencional, la duración de las ventanas de pronunciación imaginada de una palabra es variable tanto en ventanas de un sólo individuo como en ventanas de individuos distintos. Para tratar con este problema, los coeficientes wavelets son normalizados mediante la energía relativa wavelet que se describe a continuación. 
Energía wavelet relativa. Tal como se explica en [23], una vez aplicada la DWT sobre la señal se obtienen coeficientes de aproximación y de detalle, desde los cuales es posible calcular la energía relativa wavelet. La energía relativa wavelet representa la energía que algún nivel de descomposición aporta al total de la energía wavelet de la señal. La energía relativa provee información para caracterizar la distribución de energía de la señal en diferentes bandas de frecuencia, con lo que se obtiene una independencia del tamaño de la ventana de señal de EEG o de audio, según sea el caso.

A partir de la descripción anterior, en las señales EEG sonificadas se determinó usar 10 valores que representan la energía wavelet en todos los niveles de descomposición y el último de aproximación (D1-D9 y A9). Mientras que, cada ventana de habla imaginada de las señales de EEG no sonficadas se representa mediante un conjunto de 5 valores de energía wavelet, 4 de los niveles de descomposición y uno de aproximación (D2-D5 y A5) con respecto a la energía wavelet total. Tal como se realizó en [23], el valor asociado con D1 es descartado.

\subsection{Clasificación}

De acuerdo con Michie et al. [16, la clasificación cubre cualquier contexto en el que alguna decisión o pronóstico es hecho sobre la base de información histórica disponible. La base de información disponible está dada por vectores de información multi-dimensionales, cuyos valores (llamados atributos o características) pueden ser reales, discretos o nominales. El objetivo de la clasificación es inferir una relación entre un vector de datos y una posible clase (o categoría), para ello se crea un modelo que automáticamente encuentra dichas relaciones. El modelo es creado basado en una partición de entrenamiento del vector de datos. Los modelos aprendidos de los datos de entrenamiento son, entonces, evaluados con un conjunto de prueba distinto para determinar si los modelos pueden ser generalizados a nuevos casos. En el presente trabajo se entrena y prueba el clasificador Random Forest (RF) bajo un enfoque de validación cruzada usando 10 pliegues (folds).

Random forest (RF). RF es una combinación de árboles predictores tal que cada uno de los árboles depende de los valores de un vector aleatorio muestreado independientemente y con la misma distribución para todo los árboles en el bosque. Cada árbol arroja un único voto para la clase más popular para una entrada $x$ dada, y al final la salida de RF se realiza usando voto mayoritario. En [320] se describe a detalle el algoritmo de clasificación de RF incluyendo el proceso para construir los arboles individuales.

En el presente trabajo se utilizaron los siguientes hiper-parámetros para la implementación del clasificador en Weka 3.6.8: el número de árboles es 50 y el número de atributos considerados en cada nodo es $\log _{2}$ (numeroCaracteristicas) +1 . 


\section{Experimentación y resultados}

A pesar de que el EPOC de Emotiv ofrece la posibilidad de registrar 14 canales, sólo serán de interés para los experimentos los canales F7, FC5, T7 y P7. Estos canales, de acuerdo con el modelo Geschwind-Wernicke, son los más relacionadas con la producción del habla en el hemisferio izquierdo del cerebro (a excepción de algunas personas zurdas) [11.

\subsection{Selección de parámetros de la sonificación}

Dado que existen diversos parámetros en el proceso de sonificación, se tenía que elegir valores que favorecieran los porcentajes de exactitud de la clasificación, por ello para la selección de parámetros se realizó un proceso empírico de elección iterativo para cada parámetro. Es decir sólo variando un parámetro se evaluó su comportamiento de acuerdo a la exactitud de clasificación y se eligió el mejor valor, después usando ese valor se varió el siguiente parámetro y se eligió también su mejor valor, así hasta tener los mejores valores de todos los parámetros. Los valores seleccionados de los parámetros fueron: número de tonos (14), frecuencia mínima de la señal EEG $(1 \mathrm{~Hz})$, frecuencia máxima de la señal EEG $(60 \mathrm{~Hz})$, tamaño de la ventana del espectrograma(26 muestras), traslape entre ventanas del espectrograma (1 muestra), duración de los tonos (0.6 secs), frecuencia mínima del audio $(50 \mathrm{~Hz})$, frecuencia máxima del audio $(5000 \mathrm{~Hz})$, frecuencia de muestreo del audio $(8000 \mathrm{~Hz})$. En la figura 4 se muestran ejemplos de espectrogramas obtenidos usando los parámetros mencionados anteriormente.
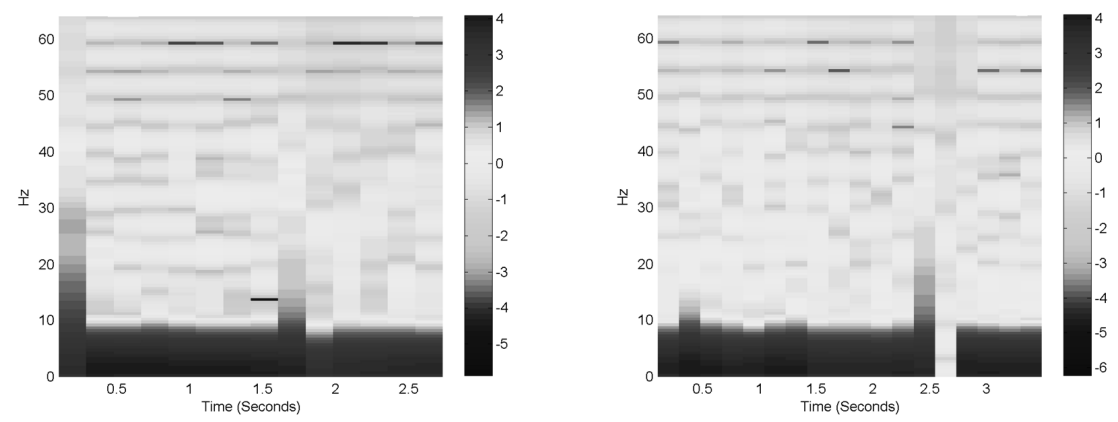

Fig. 4: Ejemplos de espectrogramas del sujeto 11, durante la pronunciación imaginada de dos distintas repeticiones de la palabra arriba, en el canal F7.

\subsection{Selección de wavelet}

En el proceso de extracción de características se siguió el mismo enfoque que en la selección de parámetros para la sonificación, se experimentó con distintos niveles de diferentes Wavelets Daubechies (db2, db6 y db20) probando una variación a la vez. La wavelet Daubechis 20 con 6 niveles fue la que mejores resultados obtuvo. 


\subsection{Resultados comparativos}

Los porcentajes de exactitud promedio al clasificar usando Random Forest para los 27 sujetos en los dos enfoques son mostrados en la figura 5.

Al analizar la tabla podemos observar que el método que sonifica la señal de EEG mejora los porcentajes de exactitud promedio en 24 de los 27 sujetos analizados, inclusive existen diferencias arriba de $15 \%$ en algunos casos. De manera general se puede concluir que el método para sonificar la señal de EEG usando el algoritmo EEG to tones permite mejorar en promedio $7.72 \%$ al conjunto de los 27 sujetos.

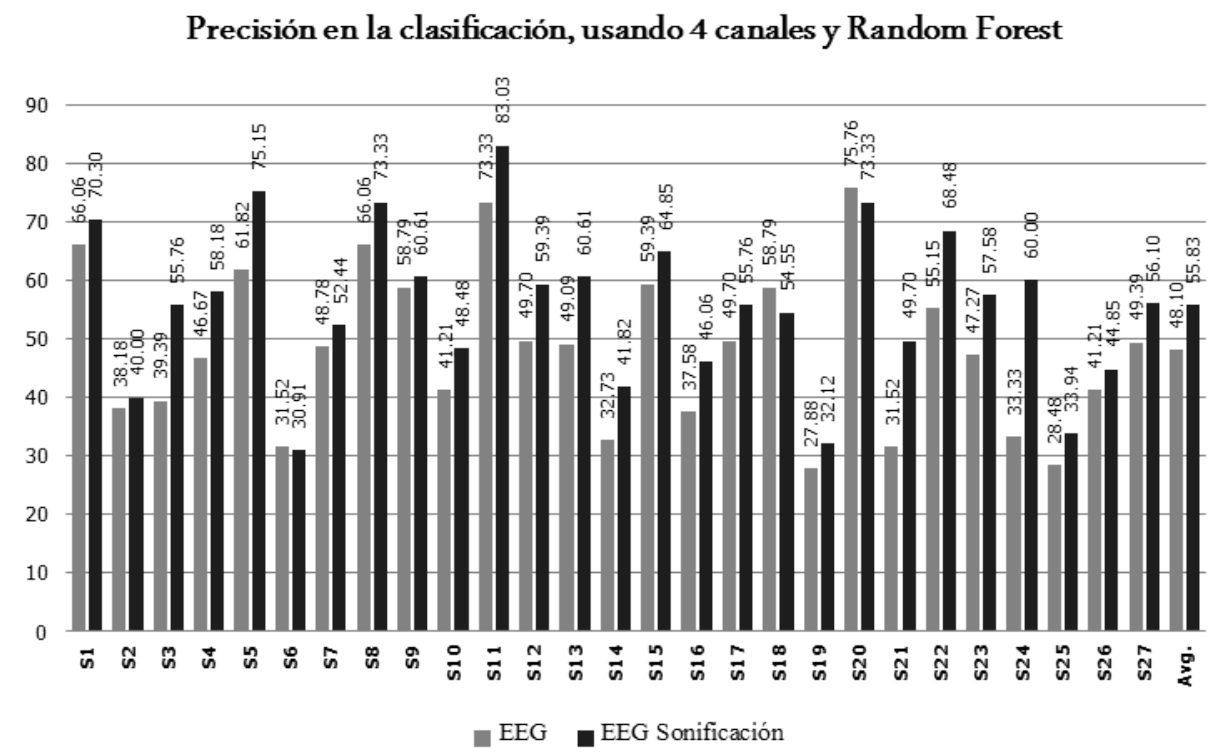

Fig. 5: Gráfica comparativa de los dos métodos EEG y Sonificación de EEG. Se muestran los porcentajes de exactitud promedio en la clasificación con Random Forest para los 27 sujetos usando 4 canales. En la ultima columna se muestran los porcentajes de clasificación promedio: EEG 48.10\% y EEG usando Sonificación $55.83 \%$.

\section{Conclusiones}

En este trabajo se realizó la clasificación de la señal de EEG aplicando una transformación de la señal original de EEG a una señal de audio, con lo cual se mejoró la exactitud promedio de clasificación en $7.72 \%$. Mediante la elección de las frecuencias dominantes del espectrograma de la señal de EEG y el mapeo de las frecuencias de EEG a frecuencias del audio, se logró resaltar patrones de la señal que ayudaron a mejorar la exactitud de la clasificación, esto ocurrió incluso 
usando la misma métodologia de extracción de características y el mismo método de clasificación, con respecto con el cual se compara. Los resultados obtenidos dan pie a realizar pruebas con otros métodos de sonificación, extracción de características y clasificación, que permitan mejorar los porcentajes de exactitud alcanzados. Un aspecto a considerar, en el trabajo por realizar, es la elección de parámetros para cualquier forma de sonificación, la cual podría ser realizada por un algoritmo de búsqueda automática, como por ejemplo un algoritmo genético. También se tendrá que calcular y comparar el comportamiento de los dos enfoques usando los 14 canales disponibles.

\section{Referencias}

1. Andersonl, C.: Eeg to tones, dept. of commputer science, colorado state university (2005)

2. Bashashati, A., Fatourechi, M., Ward, R., Birch, G.: A survey of signal processing algorithms in brain-computer interfaces based on electrical brain signals. Journal of Neural engineering 4, R32-R57 (2007)

3. Breiman, L.: Random forests. Machine learning 45(1), 5-32 (2001)

4. Brigham, K., Kumar, B.: Imagined Speech Classification with EEG Signals for Silent Communication: A Preliminary Investigation into Synthetic Telepathy. In: Bioinformatics and Biomedical Engineering (iCBBE), 2010 4th International Conference on. pp. 1-4. IEEE (2010)

5. Brouse, A., Filatriau, J.J., Gaitanis, K., Lehembre, R., Macq, B., Miranda, E., Zénon, A.: An instrument of sound and visual creation driven by biological signals. Proceedings of ENTERFACE06, Dubrovnik (Croatia).(Not peer-reviewed report.) (2006)

6. Brumberg, J.S., Nieto-Castanon, A., Kennedy, P.R., Guenther, F.H.: Brain-computer interfaces for speech communication. Speech Communication (52), 367-379 (2010)

7. DaSalla, C.S., Kambara, H., Koike, Y., Sato, M.: Spatial filtering and single-trial classification of EEG during vowel speech imagery. In: i-CREATe '09: Proceedings of the 3rd International Convention on Rehabilitation Engineering \& Assistive Technology. pp. 1-4. ACM, New York, NY, USA (2009)

8. Denby, B., Schultz, T., Honda, K., Hueber, T., Gilbert, J., Brumberg, J.: Silent speech interfaces. Speech Communication 52(4), 270-287 (2010)

9. D'Zmura, M., Deng, S., Lappas, T., Thorpe, S., Srinivasan, R.: Toward EEG sensing of imagined speech. Human-Computer Interaction. New Trends pp. 40-48 (2009)

10. Elgendi, M., Rebsamen, B., Cichocki, A., Vialatte, F., Dauwels, J.: Real-time wireless sonification of brain signals. In: Advances in Cognitive Neurodynamics (III), pp. 175-181. Springer (2013)

11. Geschwind, N.: Language and the brain. Scientific American (1972)

12. Hermann, T., Meinicke, P., Bekel, H., Ritter, H., Müller, H.M., Weiss, S.: Sonification for eeg data analysis. In: Proceedings of the 2002 International Conference on Auditory Display (2002)

13. Kramer, G., Walker, B., Bonebright, T., Cook, P., Flowers, J., Miner, N., Neuhoff, J., Bargar, R., Barrass, S., Berger, J., et al.: The sonification report: Status of the field and research agenda. report prepared for the national science foundation 
by members of the international community for auditory display. International Community for Auditory Display (ICAD), Santa Fe, NM (1999)

14. Kramer, G.: Auditory display: Sonification, audification, and auditory interfaces. Addison-Wesley Reading, MA (1994)

15. Lotte, F., Congedo, M., Lécuyer, A., Lamarche, F., Arnald, B.: A review of classication algorithms for EEG-based brain-computer interfaces. Journal of Neural Engineering 4, r1-r13 (2007)

16. Michie, D., Spiegelhalter, D., Taylor, C.: Machine Learning, Neural and Statistical Classification. Overseas Press (2009)

17. Pfurtscheller, G.: Brain-computer interfaces: State of the art and future prospects. In: Proceedings of the 12th European Signal Processing Conference: EUROSIPCO 04. pp. 509-510 (2004)

18. Pinsky, M.: Introduction to Fourier analysis and wavelets, vol. 102. Amer Mathematical Society (2002)

19. Porbadnigk, A., Schultz, T.: EEG-based Speech Recognition: Impact of Experimental Design on Performance. Master's thesis, Institut für Theoretische Informatik Universität Karlsruhe (TH), Karlsruhe, Germany (2008)

20. Rokach, L.: Pattern Classification Using Ensemble Methods. World Scientific (2009)

21. Rutkowski, T.M., Vialatte, F., Cichocki, A., Mandic, D.P., Barros, A.K.: Auditory feedback for brain computer interface management-an eeg data sonification approach. In: Knowledge-Based Intelligent Information and Engineering Systems. pp. 1232-1239. Springer (2006)

22. Torres-García, A.A., Reyes-García, C.A., Villaseñor-Pineda, L.: Toward a silent speech interface based on unspoken speech. In: Huffel, S.V., Correia, C.M.B.A., Fred, A.L.N., Gamboa, H. (eds.) BIOSTEC - BIOSIGNALS. pp. 370-373. SciTePress (2012)

23. Torres-García, A.A., Reyes-García, C.A., Villaseñor-Pineda, L.: Análisis de Señales Electroencefalográficas para la Clasificación de Habla Imaginada. Revista Mexicana de Ingeniería Biomédica 34(1), 23-39 (2013)

24. Vialatte, F., Cichocki, A., Dreyfus, G., Musha, T., Rutkowski, T.M., Gervais, R.: Blind source separation and sparse bump modelling of time frequency representation of eeg signals: New tools for early detection of alzheimer's disease. In: Machine Learning for Signal Processing, 2005 IEEE Workshop on. pp. 27-32. IEEE (2005)

25. Wand, M.: Wavelet-based Preprocessing of Electroencephalographic and Electromyographic Signals for Speech Recognition. Studienarbeit Lehrstuhl Prof. Waibel Interactive Systems Laboratories Carnegie Mellon University, Pittsburgh, PA, USA and Institut für Theoretische Informatik Universität Karlsruhe (TH), Karlsruhe, Germany (2007)

26. Wang, L., Zhang, X., Zhong, X., Zhang, Y.: Analysis and classification of speech imagery eeg for bci. Biomedical Signal Processing and Control 8(6), 901-908 (2013)

27. Wester, M., Schultz, T.: Unspoken Speech - Speech Recognition Based On Electroencephalography. Master's thesis, Institut für Theoretische Informatik Universität Karlsruhe (TH), Karlsruhe, Germany (2006)

28. Wolpaw, J., Birbaumer, N., McFarland, D., Pfurtscheller, G., Vaughan, T.: Brain-computer interfaces for communication and control. Clinical neurophysiology 113(6), 767-791 (2002) 